\title{
Legg-Calve-Perthes Disease: A Rare Cause of Bilateral Avascular Necrosis of the Hip Joints
}

\author{
*QT Islam¹, MR Siddiqui², A Hossain ${ }^{3}$, E Mustafa4, MY Rahman 5 , H Sina 6 \\ *1Prof. Dr. Quazi Tarikul Islam, Professor, Dept. of Medicine, Popular Medical College, Dhaka \\ ${ }^{2}$ Dr. Mahmudur Rahman Siddiqui, Assistant Professor, Dept. of Medicine, Anwer Khan Modern Medical College, Dhaka \\ ${ }^{3}$ Dr. Ekramul Mustafa, Indoor Medical Officer, MU-III, Dhaka Medical College Hospital, Dhaka \\ ${ }^{4}$ Dr. Ahmed Hossain, Assistant Professor, Dept. of Medicine, Dhaka Medical College Hospital, Dhaka \\ ${ }^{5}$ Dr. Md. Yousuf Ur Rahman, Asst. Registrar, MU-III, Dhaka Medical College Hospital, Dhaka \\ ${ }^{6}$ Dr. Hashmi Sina, Registrar, MU-III, Dhaka Medical College Hospital, Dhaka \\ *Corresponding Author
}

\begin{abstract}
Perthes' disease is a disorder of the hip joint in children. Femoral head became softens, flattened or deformed because of the lack of blood supply of unknown cause. It is a very rare cause of bilateral avascular necrosis of the head of the femur, which can easily diagnosed by clinical correlation with the X-ray pelvis findings. Here we present a typical case of Perthes disease, who was finally diagnosed after a long periods of time. In Perthes disease early detection is very important to preserve, restore the femoral head, relieve pain and prevent long term osteoarthritis.
\end{abstract}

Key wards: Legg-Calve-Perthes disease, avascular necrosis, femoral head

\section{Introduction}

Legg-Calvé-Perthes disease is one type of osteochondritis of the hip joint, where loss of bone mass leads to some degree of collapse of the hip joint which forms deformity of the ball of the femur and sometimes the surface of the hip socket. This disease is an orthopaedic condition that affects the blood supply of the femoral head (avascular necrosis) predominantly in children between the ages of 3 to 9 years. 1 Males are affected 4-5 times more often than females, with $10-12 \%$ of children presenting with a bilateral condition $^{2}$. It is also known as Perthes Disease, Legg-Perthes Disease, or Legg-Calve-Perthes Disease (LCPD) or syndrome, occurring in approximately 5.5 of 100,000 children per year (and therefore a lifetime risk of a child developing the disease is about 1 per 1200 individuals). Caucasians are affected more frequently than other races. 3 There is no known cause, but the literature suggests that factors such as passive smoking, maternal cigarette smoking, poverty, attention deficit hyperactivity disorder, delayed skeletal age, short stature, metabolic disorders, and mechanical dysfunction may play a role $2,3,4$.

\section{Case Report}

A 19 years old young lady reported to us with the complaints of low backache for last 6 years. Pain usually aggravated by walking and partially subsided by taking analgesic. For last 2 years she also developed occasional pain in her both knee on walking, without any swelling or redness. From very childhood she also had limping gait. Clinical examination revealed the presence of a limping or Trendelenburg gait and wasting of both quadriceps muscles. His range of movement in both hip joints in all direction was restricted, which was more pronounced during internal and abduction of both hip joints. All other systemic examination revealed normal findings.

The X-ray of the pelvis with both hip joints showed both the femoral head was deformed with short neck, irregular articular erosion with sub articular sclerosis in the both acetabulam and femoral head. Joint spaces were reduced. All features correlate with Perthes disease. Whole body scintigraphy showed increased radiotracer uptake in both hip joints and spines. With all of these findings she was diagnosed as a case of Legg-Calve-Perthes disease and referred to orthopaedic centre for better management. 


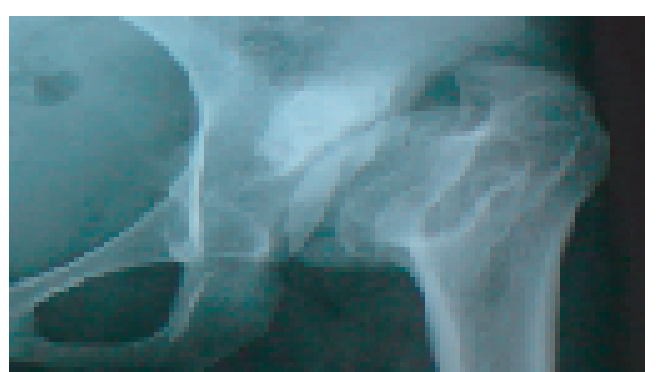

Fig 1: X-ray of the right hip joint showing deformed femoral head with short neck and irregular articular erosion with sub articular sclerosis.

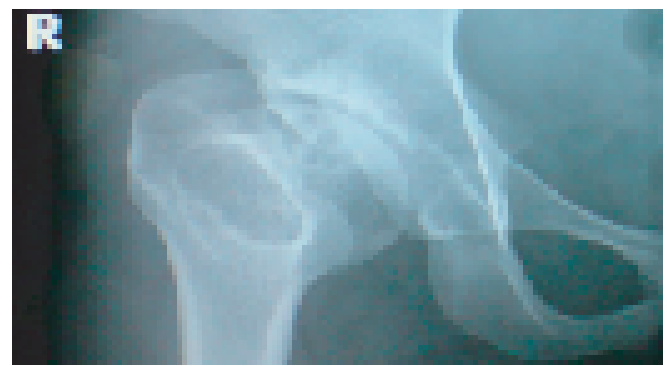

Fig 2: X-ray of the left hip joints showing same features.

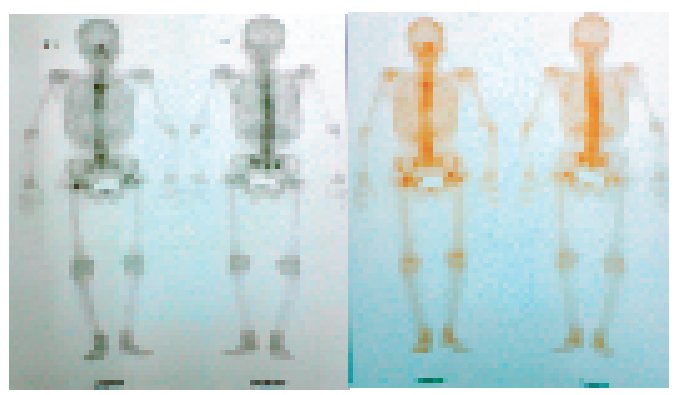

Fig 3: Scintigraphy showed increased radiotracer uptake in both hip joints and spines.

\section{Discussion}

Legg-Calvé-Perthes disease (LCPD) is an idiopathic osteonecrosis of the capital femoral epiphysis of the femoral head. Children may present with a limp and hip, knee, or groin pain with limited range of motion to the affected joint. A positive Trendelenburg gait sign can also be present, with wasting of quadriceps. The diagnosis is made with a pelvic $\mathrm{x}$-ray, which shows some degree of flattened, deformed femoral head ${ }^{2}$. Bone Scintigraphy can be done to see the extend of activity. The condition progresses over four stages (1) avascular, (2) fragmentation, (3) reossification/regeneration, and (4) healing1.
The goal of treatment is to avoid severe degenerative arthritis. Traditional treatment involves periods of bed rest, mobilization using walking aids, non-weight-bearing, abduction bracing, and surgical containment. Children under the age of 6 years usually have good results without major treatment interventions. ${ }^{4}$ Surgical containment involves a femoral, pelvic osteotomy, or shelf procedure that provides adequate coverage for the femoral head. The aim of treatment is to preserve and restore the femoral head and to relieve pain. There is debate regarding when surgical containment should be performed. It is difficult to predict the duration of the disease and the time at which revascularization of the capital femoral epiphysis occurs. A recent large trial favors surgical intervention over nonoperative management in terms of long-term outcome. 5 When treating this condition, outcomes associated with minimal femoral head deformity. Children who have been diagnosed with Perthes' Disease after the age of 10 are at a very high risk of developing osteoarthritis and Coxa Magna. 6 Conditions such as osteoarthritis requiring hip replacement in early adult years are associated with major deformities of the femoral head. Long-term concerns of developing degenerative osteoarthritis must also be considered and monitored as the child grows into adulthood 1 .

\section{Reference}

1. Maher AB, Salmond SW, Pellino TA. Alexander M. Congenital and developmental disorders. Orthopaedic Nursing 2002, 3rd ed., 567-70.

2. Bronson WE. The pediatric hip. Current Opinion in Orthopaedic, 2001; 12(6), 470-79.

3. Bahmanyar S, Montgomery SM, Weiss RJ, et al. Maternal smoking during pregnancy, other prenatal and perinatal factors, and the risk of Legg-CalvéPerthes disease. Pediatrics 2008; 122(2): e459-64.

4. Carney BT, Minter CL. Nonsurgical treatment to regain hip abduction motion in Perthes disease: A retrospective review. Southern Medical Journal 2004; 97(5), 485-88.

5. Herring JA, Kim HT, Browne R. Legg-Calve-Perthes disease. Part II: Prospective multicenter study of the effect of treatment on outcome. Journal of Bone Joint Surgery America 2004; 86-A(10), 2121-34.

6. Balasa VV, Gruppo RA, Glueck CJ, et al. LeggCalve-Perthes disease and thrombophilia. J Bone Joint Surg Am 2008; 86-A (12): 2642-7. PMID 15590848. 